\title{
S-nitrosylation: integrator of cardiovascular performance and oxygen delivery
}

\author{
Saptarsi M. Haldar ${ }^{1}$ and Jonathan S. Stamler ${ }^{1,2,3}$
}

1Department of Medicine and Cardiovascular Division, ${ }^{2}$ Harrington Discovery Institute, and ${ }^{3}$ Institute for Transformative Molecular Medicine, Case Western Reserve University School of Medicine and University Hospitals Case Medical Center, Cleveland, Ohio, USA.

\begin{abstract}
Delivery of oxygen to tissues is the primary function of the cardiovascular system. NO, a gasotransmitter that signals predominantly through protein $S$-nitrosylation to form $S$-nitrosothiols (SNOs) in target proteins, operates coordinately with oxygen in mammalian cellular systems. From this perspective, SNO-based signaling may have evolved as a major transducer of the cellular oxygen-sensing machinery that underlies global cardiovascular function. Here we review mechanisms that regulate $S$-nitrosylation in the context of its essential role in "systems-level" control of oxygen sensing, delivery, and utilization in the cardiovascular system, and we highlight examples of aberrant $S$-nitrosylation that may lead to altered oxygen homeostasis in cardiovascular diseases. Thus, through a bird's-eye view of $S$-nitrosylation in the cardiovascular system, we provide a conceptual framework that may be broadly applicable to the functioning of other cellular systems and physiological processes and that illuminates new therapeutic promise in cardiovascular medicine.
\end{abstract}

\section{Oxygen and NO: co-evolution for common function}

From the appearance of the simplest metazoans to the most complex multicellular life forms, the ability to efficiently handle oxygen has remained essential for survival and has therefore been subject to intense evolutionary pressure. A single-cell organism must rapidly adapt its core homeostatic processes to fluctuations in oxygen tension, functions retained in specialized cells of higher vertebrates $(1,2)$. In addition to cell-autonomous pathways for oxygen homeostasis, complex multicellular organisms have also developed sophisticated mechanisms to efficiently coordinate oxygen delivery and utilization across diverse organ systems (3-5). From this perspective, the human cardiovascular system in all its complexity has evolved for the principal purpose of oxygen delivery.

Although oxygen itself can function as a signaling molecule (2, $6)$, its signaling repertoire is dependent largely on heme binding and is therefore limited, as hemes do not generally convey cellular signals. Thus, organisms have necessarily evolved parallel mechanisms to precisely control oxygen flux and function. Utilization of the ancient gasotransmitter NO, highly abundant in the primordial atmosphere and linked to anaerobic respiration, likely co-evolved with oxygen to serve a common function - regulation of aerobic respiration (i.e., oxygen delivery and utilization). While $\mathrm{NO}$, like $\mathrm{O}_{2}$, binds transition metal centers to elicit cellular signals, the majority of its cellular influence is achieved through posttranslational modification (PTM) of cysteine thiols, a process termed $S$-nitrosylation (7). The universal presence of cysteine thiols in all major classes of proteins greatly expands signaling possibilities, and regulation of protein function via $S$-nitrosylation may be viewed as the prototypical system for redox-based and gasotransmitter-mediated signal transduction (8).

Recent reviews of $S$-nitrosylation have detailed the redox biochemistry of reactive nitrogen species (8-10) and cataloged the myriad proteins and cellular processes known to be regulated by this modification across systems, including the cardiovascular system (11, 12). Nearly 1,000 S-nitrosylated proteins have been identified in the

Conflict of interest: The authors have declared that no conflict of interest exists. Citation for this article: J Clin Invest. 2013;123(1):101-110. doi:10.1172/JCI62854. heart alone $(13,14)$, and cross-talk with a plethora of other PTMs has been described (15). Principles underlying reversibility, specificity, and enzymatic control of $S$-nitrosylation have received particular attention. Here, we take a thematic perspective that highlights the essential role of protein $S$-nitrosylation in the systems-level control of oxygen delivery and utilization, which is arguably the essential function of the cardiovascular system. Using these physiological insights, we highlight examples of how $S$-nitrosylation is dysregulated in cardiovascular disease and how modulation of this signaling mechanism holds therapeutic promise. Through this bird's-eye view of $S$-nitrosylation in the cardiovascular system, we provide a conceptual framework that may be broadly applicable to cellular systems, physiological processes, and diseases.

\section{S-nitrosylation as a prototypical system of protein PTM}

Systems governing PTM of proteins generally fall into two broad categories, those with a ubiquitous sphere of influence (e.g., phosphorylation) and those with a more limited cellular purview (e.g., methylation). S-nitrosylation, like phosphorylation, is clearly evolutionarily conserved and ubiquitous, affecting most, if not all classes of proteins across all cellular compartments $(8,9,16)$. By contrast, other oxygen/redox-based modifications, including hydroxylation and sulfenylation, have been identified to date with specific classes of proteins and functions $(2,17)$. Here, we draw parallels between $S$-nitrosylation and other important PTMs (e.g., phosphorylation, ubiquitinylation, acetylation) to provide a conceptual framework for understanding the molecular machinery that governs this fundamental biologic process (Figure 1).

In mammals, the principal sources of newly synthesized NO are the three NOS isoforms (NOS1-3). Nitrate and nitrite may also contribute to the NO reservoir $(18,19)$, particularly under duress. The transfer of the NO moiety to cysteine thiols in target proteins is carried out by peptide or protein nitrosylases, which mediate either metal-to-Cys or Cys-to-Cys transfer. Metal-to-Cys nitrosylases are proteins that transfer NO groups from transition metals (e.g., $\mathrm{Fe}^{2+}, \mathrm{Cu}^{2+}$ ) to cysteine thiol. For example, mammalian hemoglobin $(\mathrm{Hb})$ undergoes auto-nitrosylation via intramolecular transfer of $\mathrm{NO}$ from heme iron (iron nitrosyl; $\mathrm{HbFeNO}$ ) to a 


\section{A} Moiety S-nitrosylation

$-\mathrm{N}=\mathrm{O}$
NO group

Source NOSs (NOS $1 / 2 / 3)$
Yes Occurs across all functional classes of proteins and cellular compartments
Phosphorylation

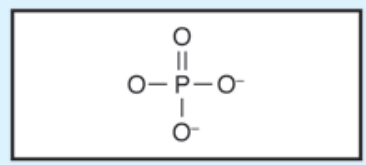

ATP
Ubiquitinylation

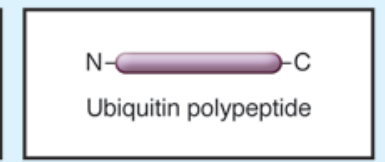

Ubiquitin
Acetylation

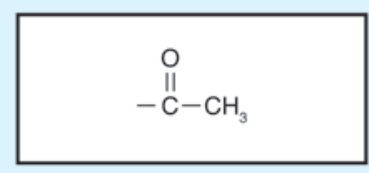

Acetyl-CoA

\section{Ubiquitous?}

Nitrosylases

Enzymatic machinery mediating transfer

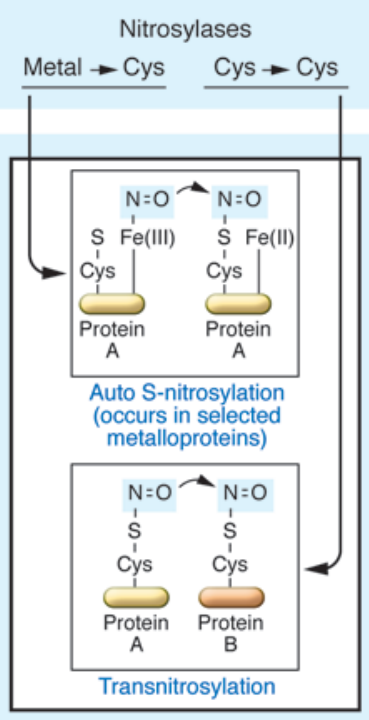
Yes
Occurs across all functional classes of proteins and cellular compartments
But with a predominance
for protein degradatory pathways

Protein kinases (Ser/Thr/Tyr)
E1 - ubiquitin-activating enzyme E2 - ubiquitin-conjugating enzyme E3 - ubiquitin ligase

\section{Transfer} biochemistry
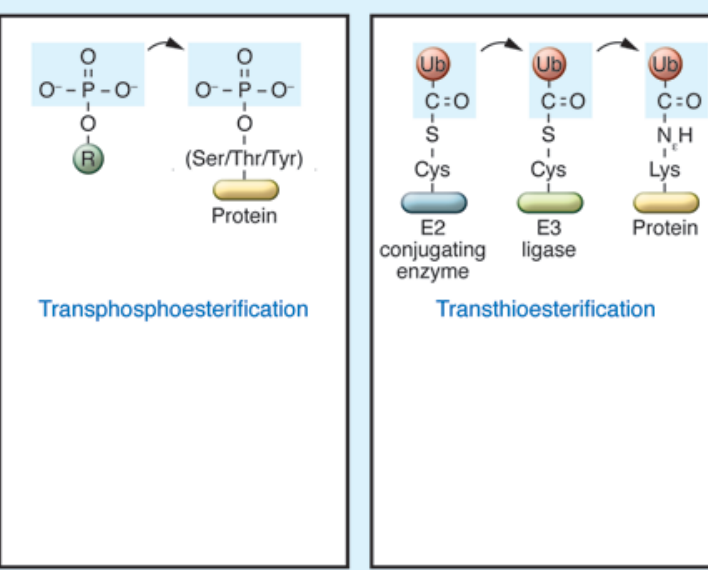

Transthioesterification

\section{Yes} in transcription and metabolic signaling
But with a dominant role

Acetyltransferase
Enzymatic machinery mediating removal

\section{Denitrosylases \\ (see panel B)}

\section{Specificity} determinants
Spatiotemporally confined interactions between substrates and sources

Enzymatic machinery

Motifs (alignment of enzymes

and specific substrate sites

and regulation of thiol $\mathrm{pK}$ )

Can be stimulus coupled

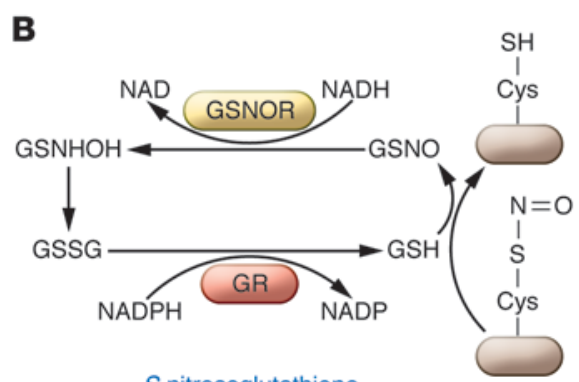

S-nitrosoglutathione reductases (GSNOR) systems

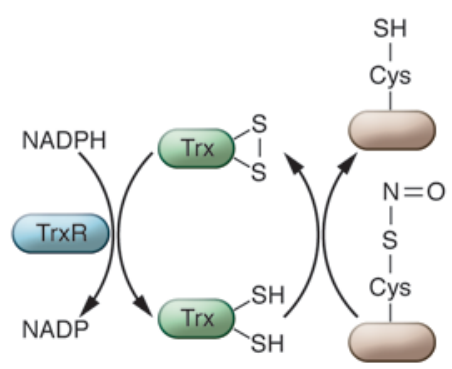

Thioredoxin systems 


\section{Figure 1}

Framework for PTMs and signaling. (A) Parallels between S-nitrosylation and other PTMs (phosphorylation, ubiquitinylation, and acetylation) highlight shared features of bona fide signaling systems. $S$-nitrosylation is ubiquitous, reversible, and subject to enzymatic control (by nitrosylases and denitrosylases), enabling spatiotemporal and target specificity. The biochemistry of NO group transfer and denitrosylation reactions is depicted. Note that auto-S-nitrosylation is shown as an example of metal-to-Cys NO transfer (e.g., as occurs from the $\mathrm{Hb}$ heme center to Cys $\beta 93$ to form SNO-Hb). However, metal-to-Cys NO transfer between two different peptides or proteins may also occur (e.g., between cytochrome $C$ and glutathione to form GSNO). (B) Denitrosylases. Two classes of denitrosylases are shown, which comprise four enzymes in mammals, including two GSNORs and two thioredoxins. GR, glutathione reductase; GSH, reduced glutathione; $\mathrm{GSNHOH}$, glutathione $\mathrm{N}$-hydroxysulfenamide; GSSG, oxidized glutathione; TrxR, Trx reductase.

specific cysteine thiol in the $\beta$-globin chain (Cys $\beta 93)(20,21)$. Similarly, transfer of metal-coordinated NO from cytochrome $c(22)$ or ceruloplasmin (23) is employed in the synthesis of $S$-nitrosoglutathione (GSNO). Cys-to-Cys nitrosylases (referred to herein as transnitrosylases) are typically $S$-nitroso-proteins (SNO-proteins) involved in Cys-to-Cys transfer of the NO group from donor to acceptor protein $(9,24)$. The net nitrosylation (NO group transfer) reaction is analogous to the group transfer reactions catalyzed by ubiquitin ligases, palmitoyltransferases, or acetyltransferases (Figure 1). Conversely, protein denitrosylation has been shown to play a major role in decreasing cellular $S$-nitrosylation (25-29) much the same way that protein phosphatases, deubiquitinases, and deacetylases set cellular levels of their respective PTMs (9, 11). To date, two major enzymatic systems mediating protein denitrosylation have been described (Figure 1): GSNO reductase (GSNOR) $(30,31)$ and thioredoxin (Trx) $(26,32)$. The GSNOR system (two GSNORs have been identified, but only one has been studied in detail; ref. 25) denitrosylates GSNO, the major low-molecular-weight SNO in mammalian cells (33). Although GSNOR acts directly only on GSNO, it governs protein $S$-nitrosylation by influencing the cellular equilibrium that is maintained by transnitrosylation reactions between SNO-proteins and $\operatorname{GSNO}(8,24)$. Studies of GSNOR-deficient organisms have demonstrated the central role of GSNOR in SNO-based signal transduction and cardiovascular function (refs. 27-29, 34, 35, and Figure 2). Members of the TRX enzyme family (TRX1 and TRX2) mediate the denitrosylation of multiple SNO-protein substrates in the cytosol and mitochondria $(25,36)$.

Studies over the past decade have established close parallels between guiding principles for S-nitrosylation (SNO-based signaling) and other PTM systems. Signals are propagated through stimulus-coupled and spatiotemporally restricted interactions within signaling complexes. The composition of SNO-based signaling complexes includes NOSs (which provide the source of $\mathrm{NO}$ ), NO group donors including GSNO and other SNO-proteins that can participate in transnitrosylation (propagation) reactions, and denitrosylases (which curtail the signals) (Figure 1). By analogy to kinases, which align with substrates through hydrophobic and ionic interactions, hydrophobic and charged amino acids surrounding substrate cysteines ("SNO motifs") may provide for alignment with nitrosylases $(8,9,37)$. Thus, SNO-protein abundance reflects regulated equilibria between $S$-nitrosylation and denitrosylation pathways, rather than rates of NO production per se. The next section, which details the role of SNO-based signaling in cardiovascular function and oxygen homeostasis, illustrates the operation of these principles under physiologic and pathophysiologic states.

\section{SNO-based signaling regulates cardiovascular performance and optimizes oxygen delivery}

Systemic oxygen delivery is largely determined by microcirculatory blood flow and, to a lesser extent, by blood $\mathrm{O}_{2}$ content, which is a function of $\mathrm{Hb} \mathrm{O}_{2}$ saturation $\left(\mathrm{SaO}_{2}\right)$ and blood $\mathrm{Hb}$ concentration. SNO-based signals regulate each of these determinants and therefore play an essential role in optimizing oxygen delivery. Furthermore, $S$-nitrosylation allows for crosstalk between NO and $\mathrm{O}_{2}$-sensing pathways to signal tissue oxygen levels and to effect changes in $\mathrm{O}_{2}$ bioavailability (5). Here, we illustrate how the SNObased system exerts coordinated effects across multiple organs to provide an integrated mechanism for sensing oxygen levels and executing molecular responses to hypoxic cues (Figure 3). Inasmuch as oxygen sensing and delivery are perturbed in all cardiovascular disease, it follows that dysregulated SNO signaling contributes to disease pathogenesis.

S-nitrosylation and myocardial performance during simulated hypoxia. Signaling via the $\beta_{2}$-adrenergic receptor $\left(\beta_{2}\right.$-AR) coordinates hypoxic adaptation across multiple organs, including the lungs (improved ventilation/perfusion matching) (38-41), skeletal muscle (hypoxic vasodilation [HVD]) (41-43), and heart (augmenting contractility) (44). Through its influence on $\beta_{2}$-AR signaling, $S$-nitrosylation may regulate hypoxic responses. The $\mathrm{G}$ protein receptor kinase 2 (GRK2), which mediates $\beta_{2}$-AR desensitization, undergoes agonist-coupled inhibitory $S$-nitrosylation in an eNOS-dependent manner (28). Absent $S$-nitrosylation, cardiac contractility (28) and peripheral vasodilation (45) decline during maintained adrenergic stimulation (28). $\beta$-Arrestin2 (a scaffolding protein that targets the $\beta_{2}$-AR for internalization via endocytosis) and dynamin (a core component of the clathrin-mediated endocytotic machinery) also undergo $S$-nitrosylation downstream of the $\beta_{2}$-AR, leading to enhanced receptor trafficking $(34,46)$. Although the precise chain of molecular events is not fully understood, it is known that GRK2, $\beta$-arrestin2, and dynamin are each complexed with eNOS, and stimulation of the $\beta_{2}$-AR leads to eNOS activation and subsequent $S$-nitrosylation of these proteins (34, 46, 47). Thus, coordinate $S$-nitrosylation events may serve to enable $\beta_{2}$-AR signaling by preventing desensitization and promoting receptor recycling to facilitate oxygen transport (cardiac output) and delivery (vasodilation). The abundance of SNO-GRK2 and SNO- $\beta$-arrestin2 is diminished in eNOS ${ }^{-/-}$mice and enhanced in GSNOR ${ }^{-1-}$ mice $(28,34)$; eNOS and GSNOR thus promote $S$-nitrosylation and denitrosylation of these proteins, respectively, through the intermediacy of GSNO. eNOS also binds dynamin (46), but whether $S$-nitrosylation is mediated directly by a transnitrosylase activity of NOS $(48,49)$ or via GSNO is not known. $G S N O R^{-1-}$ mice further exhibit increases in cardiac output under basal conditions, reflecting marked peripheral vasodilation (29) as well perhaps as pronounced myocardial angiogenesis that results from stimulatory S-nitrosylation of HIF-1 $\alpha$ under normoxic conditions (35). In addition, GSNO has direct inotropic effects (50). $G S N O R^{-/-}$mice also show constitutive increases in $\beta_{2}$-AR abundance (28), as is seen in ischemia. Collectively, then, enhanced S-nitrosylation in $\mathrm{GSNOR}^{-/-}$mice underlies hypoxia-mimetic changes throughout much of the cardiovascular system (Figure 2). Sim- 


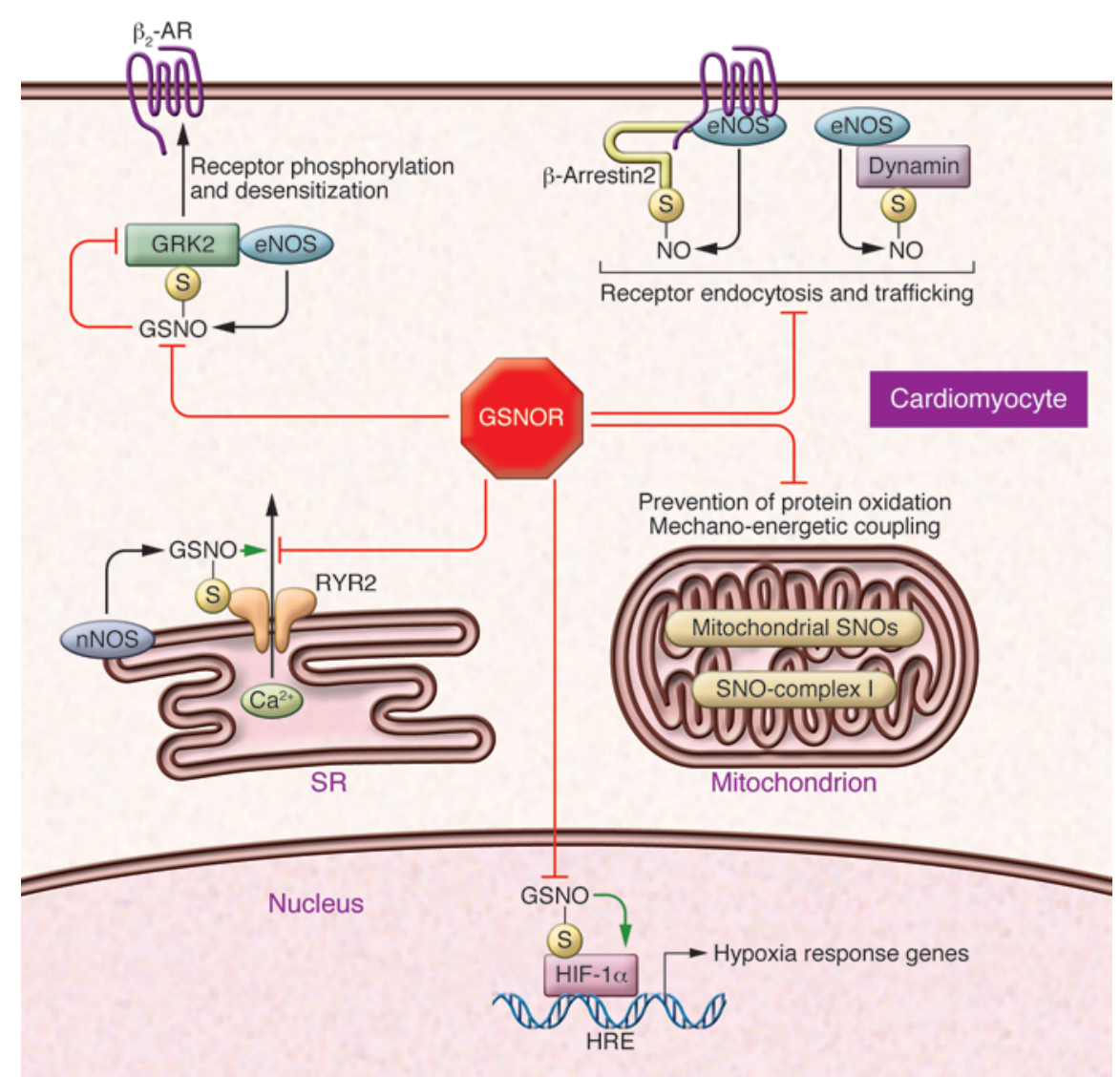

\section{Figure 2}

$S$-nitrosylation regulates cardiomyocyte signaling at critical oxygen-responsive nodal points. The central roles are highlighted for the denitrosylase GSNOR in physiologic control of $\beta_{2}$-AR signaling, SR calcium release, HIF- $1 \alpha$ responses, and mitochondrial function. S-nitrosylation reactions that have been proven by genetic criteria to occur through the intermediacy of GSNO include those targeting GRK2, RYR2, and HIF-1 $\alpha$. Ligand-dependent $S$-nitrosylation of GRK2, $\beta$-arrestin2, and dynamin is eNOS dependent. eNOS is complexed with GRK2, $\beta$-arrestin2, and dynamin, as depicted. Coordinate titration of $S$-nitrosylation (envisioned via receptor-coupled activity of transnitrosylases and denitrosylases) across multiple steps in these pathways determine net signaling responses. The effects of GSNOR (from observations in $\mathrm{GSNOR}^{-/-}$mice) manifest as increases in cardiac output under basal conditions, a persistent state of systemic vasodilation, and protection from ischemic insult, establishing a central role for GSNO in cardiovascular hemodynamics and oxygen delivery. Effects of GSNOR on mitochondrial targets are inferred from studies using GSNO. HRE, hypoxia response element. ilar SNO-based regulation of $\beta_{2}$-AR signaling in particular, and hypoxia-mimetic responses more generally, are likely operative in other tissues, including the airways and alveoli, kidney, blood, and skeletal muscle, as discussed below (Figure 3).

$S$-nitrosylation regulates striated muscle performance. Oxygen consumption can increase 5- to 10 -fold in exercising humans over the course of minutes (51). Accordingly, skeletal muscle has evolved efficient mechanisms to rapidly adapt to large shifts in oxygen demand. Just as $\beta_{2}$-AR activation increases cardiac contractility during hypoxia (44), $\beta_{2}$-AR-coupled increases in bioactive $\mathrm{NO}$ are also critical for compensatory vasodilation during mild to moderate hypoxic exercise $(52,53)$. In a manner that parallels myocardial $\beta_{2}$-AR signaling, SNO-based signals inhibit $\beta_{2}$-AR receptor desensitization in the periphery to facilitate adrenergic responses (45). Specifically, as local $\mathrm{O}_{2}$ tension $\left(\mathrm{pO}_{2}\right)$ begins to fall during exercise, NO signals to increase blood flow by potentiating $\beta_{2}$-AR-coupled HVD in working muscle (53) via a mechanism that likely involves SNO-GRK2 and inhibition of $\beta_{2}$-AR desensitization $(28,45)$. As exercise intensity and tissue hypoxia increase, the source of bioactive NO becomes less dependent upon $\beta$-adrenergic mechanisms (53) and shifts to rbc-based SNO delivery $(3,4,10,54)$ (see SNO signaling and the respiratory cycle below). Together, these two mechanisms may support oxygen delivery across a broad range of exercise intensity and duration.

A parallel mechanism operates in skeletal muscle via hypoxia-dependent, stimulatory $S$-nitrosylation of the skeletal muscle ryanodine receptor (RYR1) (55-57), a key mediator of sarcoplasmic reticulum (SR) calcium release and excitation-contraction coupling (ref. 58 and Figure 2). S-nitrosylation of RYR1 occurs only during hypoxia, increases the open probability $\left(\mathrm{P}_{\mathrm{O}}\right)$ of the channel, and potentiates SR calcium release $(57,59-62)$. This $\mathrm{pO}_{2}$-dependent SNO-RyR1 formation is mediated by NO derived from neuronal NOS (nNOS) complexed with RYR1 (63). In normoxia, by contrast, RYR1 undergoes stimulatory oxidation of redox-sensitive cysteine thiols $(55,60)$. The source of oxidizing equivalents is the SR-resident NADPH oxidase 4, which colocalizes with RYR1 and produces $\mathrm{H}_{2} \mathrm{O}_{2}$ in proportion to ambient $\mathrm{pO}_{2}$, thus functioning as a physiological oxygen sensor (55). Accordingly, both $S$-oxidation and $S$-nitrosylation stimulate RYR1, but at different physiologic $\mathrm{pO}_{2}$, reflecting conditions from resting to exercising muscle (56). Alternatively stated, when $\mathrm{pO}_{2}$ falls into the hypoxic range (as occurs in exercising muscle), regulatory thiols in RYR1 become reduced and protein conformation is allosterically altered in a manner that favors $S$-nitrosylation (57). Conversely, $S$-nitrosylation is superseded by $S$-oxidation in the normoxic conformation assumed by RYR1 in resting muscle. Thus, coordinate $S$-nitrosylation and oxidation of Cys thiols within RYR1, which are favored during hypoxia and normoxia, respectively, allow redox control over the range of physiological $\mathrm{pO}_{2}$. From a pathophysiologic perspective, excessive $S$-nitrosylation of RYR1, which can occur in settings of nitrosative stress, causes SR calcium leak and plays a maladaptive role in Duchenne muscular dystrophy (64), malignant hyperthermia (65), and exercise intolerance (66). Inasmuch as skeletal muscle dysfunction is commonly present in chronic heart failure $(67,68)$, perturbations in SNO-based signaling may underlie pathological 
crosstalk between these two tissues. Targeting key SNO-proteins common to both tissues (e.g., HDAC2) (69-72) may represent a new therapeutic approach.

Like skeletal muscle RYR1, RYR2 in cardiac muscle also undergoes nNOS-dependent stimulatory $S$-nitrosylation (refs. 60, 72, and Figure 2). However, unlike skeletal muscle (where NO can directly modify RyR1), the transfer of an NO group from nNOS to RyR2 requires GSNO (i.e., transnitrosylation) (62). In addition, SNO-RyR2 is abundant during normoxia and stimulates channel activity independently of oxygen concentration $(29,62)$. However, $\mathrm{pO}_{2}$ may retain an influence on RYR2 $S$-nitrosylation in the heart through the $\beta_{2}$-AR (see above; specifically, through $\beta_{2}$-AR-coupled RYR2 denitrosylation involving GSNOR) (29). The importance of this regulatory pathway has been established by study of GSNOR ${ }^{-/-}$mice, which exhibit depressed $\beta$-adrenergic inotropic responses, impaired $\beta$-agonist-induced denitrosylation of RYR2, and pathological calcium leak (29). Similarly, $n \mathrm{NOS}^{-/-}$hearts have diminished SNO-RyR2, excessive diastolic SR calcium leak, contractile dysfunction, and susceptibility to arrhythmias $(73,74)$. We note that while the $\beta_{2}$-AR system and RYR2 serve as important examples of SNO-based regulation, $S$-nitrosylation likely controls other aspects of cardiac homeostasis in an oxygen-dependent manner. For example, emergent evidence suggests $S$-nitrosylation of mitochondrial proteins may protect against myocardial ischemia (75), potentially via prevention of pathologic protein oxidation and inhibition of apoptosis. Likewise, inhibitory $S$-nitrosylation of mitochondrial complex I, in certain contexts, may play an adaptive role in mechanoenergetic coupling (ref. 76 and Figure 2). As disruption of the $\mathrm{SNO} /$ redox balance in myocytes is a hallmark of human heart failure (77), restoration of this equilibrium may provide a fruitful approach to restoring cardiac performance.

SNO signaling and the respiratory cycle. Oxygen delivery is a function of blood $\mathrm{O}_{2}$ content and blood flow. The ability to augment blood $\mathrm{O}_{2}$ content is markedly constrained, varying linearly with $\mathrm{Hb}$ concentration and $\mathrm{SaO}_{2}$. Conversely, modulation of regional blood flow, which is proportional to vessel radius to the fourth power, has a dynamic range encompassing several orders of magnitude. Thus, volume and distribution of local blood flow are the principal determinants of tissue oxygen delivery (10). Mammals have a robust capacity to autoregulate systemic blood flow to dynamically couple local oxygen demand with oxygen delivery - a process termed HVD. The central role of $\mathrm{rbc}$ in HVD was established half a century ago by Guyton (78), who showed that HVD is inversely proportional to $\mathrm{SaO}_{2}$ and recapitulated by rbc containing desaturated but not saturated $\mathrm{Hb}$ (78). By contrast, HVD is independent of arterial $\mathrm{pO}_{2}$ (79-81). Guyton proposed that erythrocytes sequestered a vasoconstrictor in the lungs (78), and the critical importance of $\mathrm{SaO}_{2}$ (as distinguished from $\mathrm{pO}_{2}$ ) was overlooked at the time. Later, rbc were appreciated to liberate vasodilator SNOs during hypoxia. Specifically, circulating rbc transport bioactive NO to the peripheral microcirculation and release it in proportion to locally declining oxygen gradients, in a process governed by changes in the quaternary conformation of $\mathrm{Hb}$ associated with changes in $\mathrm{O}_{2}$ concentration $(3,4,10,54,81)$. The molecular basis for this effect involves a critical cysteine within the $\mathrm{Hb}$ $\beta$-chain (Cys $\beta 93)$ that exhibits dynamic $S$-nitrosylation coupled to $\mathrm{Hb}$ allostery $(3,4,20,81)$. Oxygen binding to the heme-iron of $\mathrm{Hb}$ promotes a transition from $\mathrm{T}$ state (in deoxygenated blood) to $\mathrm{R}$ state (in oxygenated blood), during which heme-bound $\mathrm{NO}$ is transferred to the thiol group of Cys $\beta 93$. This auto- $S$-nitrosylated cysteine remains hydrophobically buried in the $\mathrm{R}$ configuration and thus devoid of vasodilatory activity. With the transition from $\mathrm{R}$ to $\mathrm{T}$ state as erythrocytes travel to increasingly hypoxic regions of the systemic microcirculation, the NO group on Cys $\beta 93$ is exposed to solvent and is released via transnitrosylative transfer to glutathione or thiols of the rbc membrane protein AE- 1 to form GSNO and SNO AE-1 $(10,54,82)$. In this manner, oxygen itself serves as a principal allosteric regulator that couples physiological release of $\mathrm{O}_{2}$ and bioactive NO. Inasmuch as blood flow is the principal determinant of $\mathrm{O}_{2}$ delivery, this remarkable function of $\mathrm{Hb}$ represents an elegant means of dynamically matching vasomotor tone with local oxygen gradients (refs. 5, 80, and Figure 2). The physiologic importance of SNO-Hb in human hypoxic adaptation was recently demonstrated in an observational study of healthy subjects undergoing progressive high-altitude acclimatization in the Himalayas (83). Blood concentrations of SNO-Hb progressively increased with ascent and were independently correlated with exercise capacity at high altitude.

SNO signaling and pulmonary gas exchange. NO bioactivity exerts control over ventilation-perfusion (V/Q) matching through a dual mechanism: (a) a permissive action on the $\beta_{2}$-AR $(28,34)$ (see above), which may improve $\mathrm{V} / \mathrm{Q}$ matching by enhancing alveolar clearance of fluid (84) and (b) the process of hypoxic pulmonary vasoconstriction (HPV), whereby the pulmonary arterial microcirculation preferentially perfuses well-ventilated alveolar units (refs. 5, 10, and Figure 3). Physiological trapping of NO by erythrocytes involves capture or inactivation of $\mathrm{NO}$ by hemes of $\mathrm{Hb}$ and serves as an important contributor to HPV (85-88). NO trapping during hypoxia may be facilitated by regulation of $\mathrm{rbc}$ membrane NO permeability via conformation-dependent binding of $\mathrm{Hb}$ to the $\mathrm{rbc}$ transmembrane protein $\mathrm{AE}-1$ (54). In normoxia, the rbc plasma membrane constitutes a significant barrier to NO entry mediated by tight association between the submembrane cytoskeleton and the cytoplasmic domain of AE-1. In hypoxia, Hb binds AE-1 (binding is favored in the T state) and alters the submembrane cytoskeletal scaffold in a manner that increases NO permeability, thereby facilitating NO trapping $(54,89-91)$. As basal vasodilatory tone in the pulmonary arterial circulation is set by a relatively high level of local NO production (from eNOS), NO trapping during hypoxia provides an important braking mechanism on vasodilation and, consequently, regional pulmonary blood flow. In other words, avid NO trapping by less-well-oxygenated erythrocytes perfusing less-well-ventilated lung units, and attenuation of NO trapping by well-oxygenated erythrocytes perfusing well-ventilated lung units, can facilitate $\mathrm{V} / \mathrm{Q}$ matching (5). $\mathrm{pO}_{2}$-regulated $\mathrm{NO}$ permeability may also facilitate unloading of bioactive $\mathrm{NO}$ from $\mathrm{SNO}-\mathrm{Hb}$ in the transition from $\mathrm{R}$ state to $\mathrm{T}$ state in the systemic microcirculation to mediate HVD and in the lungs to mitigate excessive pulmonary vasoconstriction (refs. 10, 53, and Figure 3).

Defects in NO processing by rbc are associated with multiple cardiovascular diseases, including sepsis (excess levels of SNOs in rbc) and pulmonary arterial hypertension $(\mathrm{PAH})$ (decreased rbc SNO levels). In sepsis, uncontrolled production of SNOs $(27,82,92)$, known as nitrosative stress, is believed to contribute to multiorgan failure with resultant disruption of NO-based vascular autoregulation, particularly $\mathrm{V} / \mathrm{Q}$ matching in the lung and shunting in tissues $(27,82,93)$. SNO content is increased 20 -fold in rbc from humans with septic shock and acute respiratory distress syndrome $(27,82)$, and vasoactivity of these $\mathrm{rbc}$ is dysregulated in a murine lung bioassay $(82,93)$. The link between $\mathrm{pO}_{2}$ and SNO delivery 


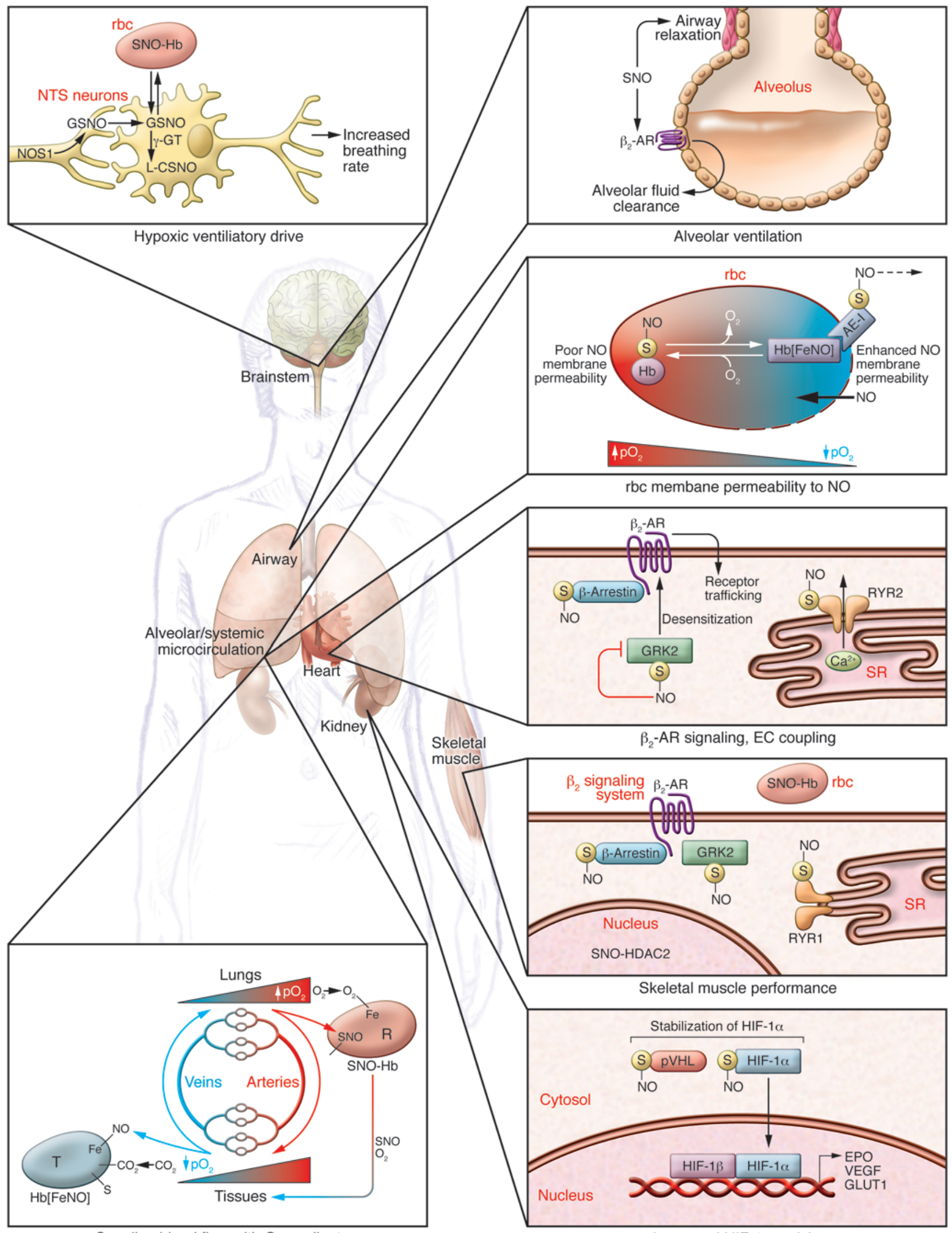

Coupling blood flow with $\mathrm{O}_{2}$ gradient

Increased HIF-1 $\alpha$ activity 


\section{Figure 3}

SNO-based integration of oxygen utilization and homeostasis across organ systems. SNO-based signals exert coordinated effects across multiple organ systems to provide an integrated mechanism for sensing oxygen levels and executing molecular responses to hypoxic cues. The roles of SNOs in cardiac and skeletal muscle performance; respiratory cycle functions (vasodilator and vasoconstrictor function of $\mathrm{Hb}$ ), including HVD and alveolar ventilation and perfusion matching (rbc NO permeability and trapping); the central ventilatory drive; and chronic adaptation to hypoxemia and anemia (HIF-1 $\alpha$ signaling) are depicted. SNO-mediated activation of HIF- $1 \alpha$ has been demonstrated in multiple tissues, including the kidney (127). Specific details shown in the kidney inset are derived, in part, from observations in the heart, brain, and other tissues. EPO, erythropoietin.

that underlies HVD is also overwhelmed in sepsis $(94,95)$, possibly due to promiscuous transnitrosylation of exofacial rbc membrane proteins that results in $\mathrm{pO}_{2}$-independent vasodilation. Accumulation of rbc SNOs and loss of allosteric control of SNO release (93, 95) may help explain the severely dysregulated blood flow pattern, which is greatly enhanced but chaotic, in the septic microcirculation. This mechanism is supported by studies of $\mathrm{GSNOR}^{-/-}$mice, which exhibit increased rbc SNO content, decreased basal vascular tone (29), and excessive mortality during experimental models of sepsis (27). Conversely, rbc in patients with $\mathrm{PAH}$ and hypoxemia have reduced levels of SNO-Hb but preserved rbc NO trapping, which reduces microcirculatory NO bioavailability. This defect may promote excessive pulmonary vasoconstriction in well-ventilated alveolar units and impair blood flow to hypoxic tissues in the systemic circulation (96). As aberrant vascular autoregulation in both the pulmonary and systemic circulation are hallmarks of advanced heart failure (97), defects in SNO processing by rbc in heart failure (98) likely also contribute to disease progression.

SNOs regulate ventilation. During hypoxia, mammals increase total lung ventilation by augmenting breathing rate (hypoxic ventilatory drive) and tidal volume, both of which are regulated by SNO-based signals. The central limb in this response is classically initiated by hypoxia-sensing cells in the carotid body, which relay to nNOS-rich neurons in the brainstem nucleus tractus solitarius (nTS). nNOS activation in the nTS is critical for the hypoxic ventilatory response and likely involves formation of low-molecular-weight SNOs (99, 100). Injection of low-molecular-weight SNOs, in particular GSNO or S-nitroso-L-cysteine, into rodent nTS dramatically increases minute ventilation in a manner that closely mimics the physiological effects of hypoxia (101). Furthermore, nTS injection of a lowmass fraction derived from deoxygenated blood, which contains $\mathrm{Hb}$-derived SNOs, reproduces the effects of GSNO, whereas a lowmass fraction derived from oxygenated blood has no effect. Using pharmacological and genetic approaches, it was also discovered that enzymatic processing of GSNO to cysteinylGlySNO by $\gamma$-glutamyl transpeptidase $(\gamma-\mathrm{GT})$ was required for GSNO to augment minute ventilation, and mice deficient in $\gamma$-GT were shown to have a grossly abnormal ventilatory response to hypoxia (101). Together, these data show that endogenous SNOs (likely those derived from deoxygenated $\mathrm{rbc}$ ) can act at the level of the nTS to mediate the ventilatory response to hypoxia (101) and SNO-based signaling may play a more pervasive role in controlling the drive to breathe, e.g., in carotid body chemoreceptors. In addition to central effects, SNOs can also augment ventilation via bronchodilation $(102,103)$ and possibly via effects on contractile function of breathing muscles (e.g., diaphragm, intercostals) $(55,56)$. These findings also suggest a link between aberrancies in SNO signaling (104) and the disrupted breathing pattern/mechanics $(104,105)$ that are frequently observed in patients with heart failure.

SNOs and the cellular response to bypoxia versus anemia. Systemic hypoxia (decreased $\mathrm{pO}_{2}$ ) and anemia (decreased rbc mass and blood $\mathrm{Hb}$ concentration) both result in reduced oxygen delivery to tissues. Although both stressors activate HIF- $1 \alpha$, a ubiquitous transcriptional regulator of hypoxic adaptation (1), the mechanism of activation differs. In sustained hypoxia, arterial $\mathrm{O}_{2}$ delivery is reduced due to low $\mathrm{pO}_{2}$ and $\mathrm{SaO}_{2}$. In this setting, inactivation of cellular $\mathrm{O}_{2}$ sensors (in particular, $\mathrm{O}_{2}$-dependent prolyl hydroxylases) results in the stabilization/accumulation of HIF- $1 \alpha$ and enhanced transcriptional activity. Canonical HIF-1 $\alpha$ targets include erythropoietin, VEGF, and GLUT1, which regulate erythropoiesis, angiogenesis, and glucose utilization, respectively $(1,5)$. On the other hand, anemia reduces blood $\mathrm{O}_{2}$ content through a reduction in $\mathrm{Hb}$ concentration while preserving $\mathrm{PaO}_{2}$ and $\mathrm{SaO}_{2}$. Therefore, while both hypoxia and anemia are associated with reduced $\mathrm{O}_{2}$ delivery, the relative sparing of $\mathrm{SaO}_{2}$ during anemia fails to trigger classical $\mathrm{O}_{2}$-dependent HIF- $1 \alpha$ signaling (5). Rather, HIF- $1 \alpha$ activation during anemia occurs because $S$-nitrosylation - generating SNOPVHL, SNO-PHD2, and SNO-HIF- $1 \alpha$ - serves to activate HIF-1 $\alpha$ under normoxic conditions (106-109). In addition, GSNOR abundance has been found to decrease in rodent models of acute anemia, which could further augment SNO bioactivity and hypoxic adaptation (110). Notably, endogenous SNOs are critical for ischemic cardioprotection in mouse models (35). Inasmuch as anemia is a robust predictor of adverse outcomes in patients with ischemic heart disease (111-113) and heart failure (114), these experimental data strongly suggest that aberrant SNO-based signals can mediate the detrimental effects of anemia in these clinical settings and suggest new therapeutic approaches.

\section{Therapeutic potential of modulating S-nitrosylation}

Decreased levels and/or impaired bioavailability of SNO-modified proteins have been observed in a variety of disease states characterized by tissue hypoxia (5), including congestive heart failure (98). To the extent that NO donors (e.g., isosorbide dinitrate, nitroglycerin) have beneficial effects in patients with heart failure and ischemic heart disease (115-118), these therapies may function, in part, via modulating $S$-nitrosylation of key myocardial proteins (e.g., RYR2, HIF- $1 \alpha$, and $\beta_{2}$-AR signaling components including GRK2). More efficient methods of SNO delivery to the myocardium (e.g., low-molecular-weight SNOs, SNO-loaded $\mathrm{rbc}$ ) may have clinical benefits in this setting (50). In particular, rbc represent an attractive vehicle for SNO delivery: (a) rbc interface with endothelium to form the largest aggregate intercellular interaction in the human body and therefore can exert beneficial effects across multiple organs; (b) rbc deliver both $\mathrm{NO}$ bioactivity and oxygen under the control of $\mathrm{pO}_{2}$-based allostery and thereby facilitate autoregulatory control (3); and (c) rbc provide unique access to the microcirculation that is dysfunctional in many cardiovascular diseases. The importance of rbc-mediated SNO delivery is reflected in the clinical observation that anemia is strongly associated with adverse outcome in patients with cardiovascular disease, even with modest levels of blood loss (111-114). Paradoxically, liberal restoration of plasma $\mathrm{Hb}$ concentration via standard clinical practices (i.e., transfusion of banked erythrocytes, administration of erythro- 
poietin) has failed to demonstrate improved tissue oxygenation or clinical benefit in this setting, and multiple studies indicate that this practice might actually be harmful (119-122). One contributory explanation for this apparent paradox is that banked blood is depleted of SNO and fails to deliver bioactive NO, and can therefore exacerbate tissue hypoxia (123-125). Accordingly, strategies aimed at restoring SNO levels in $\mathrm{rbc}(96,126)$ may represent an attractive strategy to improve NO delivery, myocardial performance, and tissue oxygenation.

\section{Conclusions}

$\mathrm{O}_{2}$ sensing and coupling mechanisms at the cellular, tissue, and integrated system levels involve critical roles for $S$-nitrosylation-based signaling, a ubiquitous and evolutionarily conserved mechanism for control of cellular function. In particular, SNOs have been shown to regulate the activities of ion channels, receptors, respiratory proteins, and enzymes that ultimately transduce hypoxic signals into increased alveolar ventilation, matched alveolar perfusion, augmentation of cardiac and skeletal muscle performance, and enhanced microcirculatory blood flow. Dysregulation of the cardiovascular system in heart failure can be understood in these terms to represent global impairments of $\mathrm{O}_{2}$ delivery, equated with reduced bioavailability of SNOs and their hypoxia-mimetic signaling function. Increased understanding of how SNO-based signals execute control of myocardial, skeletal, vascular, and hematologic function will continue to pave the way for new cardiovascular therapeutics.

\section{Acknowledgments}

This work was supported by the NIH (grants HL075443, HL095463, and AI080633 to J.S. Stamler; grant HL086614 to S.M. Haldar) and the Defense Advanced Research Projects Agency (grant N66001-10-C-2015 to J.S. Stamler).

Address correspondence to: Jonathan S. Stamler, 2103 Cornell Road, Room 5-542, Cleveland, Ohio 44106, USA. Phone: 216.368.5726; Fax: 216.368.2968; E-mail: jonathan.stamler@ case.edu. Or to: Saptarsi M. Haldar, 2103 Cornell Road, Room 4-525, Cleveland, Ohio 44106, USA. Phone: 216.368.3581; Fax: 216.368.0556; E-mail: saptarsi.haldar@case.edu.
1. Semenza GL. Hypoxia-inducible factors in physiology and medicine. Cell. 2012;148(3):399-408.

2. Kaelin WG Jr, Ratcliffe PJ. Oxygen sensing by metazoans: the central role of the HIF hydroxylase pathway. Mol Cell. 2008;30(4):393-402.

3. McMahon TJ, et al. Nitric oxide in the human respiratory cycle. Nat Med. 2002;8(7):711-717.

4. Stamler JS, et al. Blood flow regulation by S-nitrosohemoglobin in the physiological oxygen gradient. Science. 1997;276(5321):2034-2037.

5. Ho JJ, Man HS, Marsden PA. Nitric oxide signaling in hypoxia. J Mol Med (Berl). 2012;90(3):217-231.

6. Janssen-Heininger YM, et al. Redox-based regulation of signal transduction: principles, pitfalls, and promises. Free Radic Biol Med. 2008;45(1):1-17.

7. Stamler JS, et al. S-nitrosylation of proteins with nitric oxide: synthesis and characterization of biologically active compounds. Proc Natl Acad Sci US A. 1992;89(1):444-448.

8. Hess DT, et al. Protein S-nitrosylation: purview and parameters. Nat Rev Mol Cell Biol. 2005;6(2):150-166.

9. Anand P, Stamler JS. Enzymatic mechanisms regulating protein $S$-nitrosylation: implications in health and disease. J Mol Med (Berl). 2012;90(3):233-244.

10. Doctor A, Stamler JS. Nitric oxide transport in blood: a third gas in the respiratory cycle. In: Comprehensive Physiology. Vol. 1, Iss. 1. Hoboken, New Jersey, USA: John Wiley and Sons; 2011:541-568.

11. Lima B, Forrester MT, Hess DT, Stamler JS. S-nitrosylation in cardiovascular signaling. Circ Res. 2010;106(4):633-646

12. Foster MW, Hess DT, Stamler JS. Protein S-nitrosylation in health and disease: a current perspective. Trends Mol Med. 2009;15(9):391-404.

13. Kohr MJ, et al. Characterization of potential S-nitrosylation sites in the myocardium. Am J Physiol Heart Circ Physiol. 2011;300(4):H1327-H1335.

14. Kohr MJ, et al. Simultaneous measurement of protein oxidation and S-nitrosylation during preconditioning and ischemia/reperfusion injury with resin-assisted capture. Circ Res. 2011;108(4):418-426

15. Hess DT, Stamler JS. Regulation by S-nitrosylation of protein post-translational modification. J Biol Chem. 2012;287(7):4411-4418.

16. Seth D, Hausladen A, Wang YJ, Stamler JS. Endogenous protein S-Nitrosylation in E. coli: regulation by OxyR. Science. 2012;336(6080):470-473.

17. Finkel T. Signal transduction by reactive oxygen species. J Cell Biol. 2011;194(1):7-15.

18. Luchsinger BP, Rich EN, Gow AJ, Williams EM,
Stamler JS, Singel DJ. Routes to S-nitroso-hemoglobin formation with heme redox and preferential reactivity in the beta subunits. Proc Natl Acad SciU S A. 2003;100(2):461-466.

19. Lundberg JO, et al. Nitrate and nitrite in biology, nutrition and therapeutics. Nat Chem Biol. 2009;5(12):865-869.

20. Jia L, Bonaventura C, Bonaventura J, Stamler JS. S-nitrosohaemoglobin: a dynamic activity of blood involved in vascular control. Nature. 1996;380(6571):221-226.

21. Gow AJ, Stamler JS. Reactions between nitric oxide and haemoglobin under physiological conditions. Nature. 1998;391(6663):169-173.

22. Basu S, et al. A novel role for cytochrome c: Efficient catalysis of S-nitrosothiol formation. Free Radic Biol Med. 2010;48(2):255-263.

23. Inoue $\mathrm{K}$, et al. Nitrosothiol formation catalyzed by ceruloplasmin. Implication for cytoprotective mechanism in vivo. J Biol Chem. 1999;274(38):27069-27075.

24. Stamler JS, Hess DT. Nascent nitrosylases. Nat Cell Biol. 2010;12(11):1024-1026.

25. Benhar M, Forrester MT, Stamler JS. Protein denitrosylation: enzymatic mechanisms and cellular functions. Nat Rev Mol Cell Biol. 2009;10(10):721-732.

26. Benhar M, Forrester MT, Hess DT, Stamler JS. Regulated protein denitrosylation by cytosolic and mitochondrial thioredoxins. Science. 2008;320(5879):1050-1054.

27. Liu L, et al. Essential roles of S-nitrosothiols in vascular homeostasis and endotoxic shock. Cell. 2004;116(4):617-628.

28. Whalen EJ, et al. Regulation of beta-adrenergic receptor signaling by S-nitrosylation of G-protein-coupled receptor kinase 2. Cell. 2007;129(3):511-522.

29. Beigi F, et al. Dynamic denitrosylation via $S$-nitrosoglutathione reductase regulates cardiovascular function. Proc Natl Acad Sci U S A. 2012;109(11):4314-4319.

30. Jensen DE, Belka GK, Du Bois GC. S-Nitrosoglutathione is a substrate for rat alcohol dehydrogenase class III isoenzyme. Biochem J. 1998;331:659-668.

31. Liu L, Hausladen A, Zeng M, Que L, Heitman J, Stamler JS. A metabolic enzyme for S-nitrosothiol conserved from bacteria to humans. Nature. 2001;410(6827):490-494.

32. Sengupta R, Ryter SW, Zuckerbraun BS, Tzeng E, Billiar TR, Stoyanovsky DA. Thioredoxin catalyzes the denitrosation of low-molecular mass and protein S-nitrosothiols. Biochemistry. 2007; 46(28):8472-8483.
33. Bateman RL, Rauh D, Tavshanjian B, Shokat KM. Human carbonyl reductase 1 is an S-nitrosoglutathione reductase. J Biol Chem. 2008; 283(51):35756-35762.

34. Ozawa K, et al. S-nitrosylation of beta-arrestin regulates beta-adrenergic receptor trafficking. $\mathrm{Mol}$ Cell. 2008;31(3):395-405.

35. Lima B, et al. Endogenous S-nitrosothiols protect against myocardial injury. Proc Natl Acad Sci U S A. 2009;106(15):6297-6302.

36. Wu C, et al. Distinction of thioredoxin transnitrosylation and denitrosylation target proteins by the ICAT quantitative approach. J Proteomics. 2011;74(11):2498-2509.

37. Seth D, Stamler JS. The SNO-proteome: causation and classifications. Curr Opin Chem Biol. 2011;15(1):129-136.

38. Snyder EM, Johnson BD, Joyner MJ. Genetics of beta2-adrenergic receptors and the cardiopulmonary response to exercise. Exerc Sport Sci Rev. 2008;36(2):98-105.

39. Que LG, et al. Protection from experimental asthma by an endogenous bronchodilator. Science. 2005;308(5728):1618-1621.

40. Berthiaume Y, Matthay MA. Alveolar edema fluid clearance and acute lung injury. Respir Physiol Neurobiol. 2007;159(3):350-359.

41. Baloglu E, Reingruber T, Bärtsch P, Mairbäurl H. $\beta 2$-Adrenergics in hypoxia desensitize receptors but blunt inhibition of reabsorption in rat lungs. Am J Respir Cell Mol Biol. 2011;45(5):1059-1068.

42. Weisbrod CJ, Minson CT, Joyner MJ, Halliwill JR. Effects of regional phentolamine on hypoxic vasodilatation in healthy humans. J Physiol. 2001; 537(pt 2):613-621.

43. Xie L, et al. Oxygen-regulated beta(2)-adrenergic receptor hydroxylation by EGLN3 and ubiquitylation by pVHL. Sci Signal. 2009;2(78):ra33.

44. Naghshin J, et al. Chronic intermittent hypoxia increases left ventricular contractility in C57BL/6J mice. J Appl Physiol. 2009;107(3):787-793.

45. Whalen EJ, Johnson AK, Lewis SJ. Beta-adrenoceptor dysfunction after inhibition of NO synthesis. Hypertension. 2000;36(3):376-382.

46. Wang G, Moniri NH, Ozawa K, Stamler IS, Daaka Y. Nitric oxide regulates endocytosis by S-nitrosylation of dynamin. Proc Natl Acad Sci U S A. 2006;103(5):1295-1300.

47. Liu S, Premont RT, Kontos CD, Zhu S, Rockey DC. A crucial role for GRK2 in regulation of endothelial cell nitric oxide synthase function in portal hyper- 
tension. Nat Med. 2005;11(9):952-958.

48. Smith BC, Fernhoff NB, Marletta MA. Mechanism and kinetics of inducible nitric oxide synthase auto-S-nitrosation and inactivation. Biochemistry. 2012;51(5):1028-1040.

49. Ravi K, Brennan LA, Levic S, Ross PA, Black SM. S-nitrosylation of endothelial nitric oxide synthase is associated with monomerization and decreased enzyme activity. Proc Natl Acad Sci U S A. 2004;101(8):2619-2624.

50. Rassaf T, et al. Positive effects of nitric oxide on left ventricular function in humans. Eur Heart J. 2006;27(14):1699-1705.

51. Arena R, Sietsema KE. Cardiopulmonary exercise testing in the clinical evaluation of patients with heart and lung disease. Circulation. 2011;123(6):668-680.

52. Casey DP, Joyner MJ. Local control of skeletal muscle blood flow during exercise: influence of available oxygen. J Appl Physiol. 2011;111(6):1527-1538.

53. Casey DP, Curry TB, Wilkins BW, Joyner MJ. Nitric oxide-mediated vasodilation becomes independent of beta-adrenergic receptor activation with increased intensity of hypoxic exercise. J Appl Physiol. 2011;110(3):687-694.

54. Pawloski JR, Hess DT, Stamler JS. Export by red blood cells of nitric oxide bioactivity. Nature. 2001;409(6820):622-626.

55. Sun QA, et al. Oxygen-coupled redox regulation of the skeletal muscle ryanodine receptor-Ca2+ release channel by NADPH oxidase 4. Proc Natl Acad Sci U S A. 2011;108(38):16098-16103.

56. Eu JP, et al. Concerted regulation of skeletal muscle contractility by oxygen tension and endogenous nitric oxide. Proc Natl Acad Sci U S A. 2003;100(25):15229-15234.

57. Sun J, Xu L, Eu JP, Stamler JS, Meissner G. Nitric oxide, NOC-12, and S-nitrosoglutathione modulate the skeletal muscle calcium release channel/ ryanodine receptor by different mechanisms. An allosteric function for $\mathrm{O} 2$ in S-nitrosylation of the channel. J Biol Chem. 2003;278(10):8184-8189.

58. Bers DM. Cardiac excitation-contraction coupling. Nature. 2002;415(6868):198-205.

59. Sun J, Xin C, Eu JP, Stamler JS, Meissner G. Cysteine-3635 is responsible for skeletal muscle ryanodine receptor modulation by NO. Proc Natl Acad Sci U S A. 2001;98(20):11158-11162.

60. Eu JP, Sun J, Xu L, Stamler JS, Meissner G. The skeletal muscle calcium release channel: coupled $\mathrm{O} 2$ sensor and $\mathrm{NO}$ signaling functions. Cell. 2000;102(4):499-509.

61. Xu L, Eu JP, Meissner G, Stamler JS. Activation of the cardiac calcium release channel (ryanodine receptor) by poly-S-nitrosylation. Science. 1998;279(5348):234-237.

62. Sun J, Yamaguchi N, Xu L, Eu JP, Stamler JS, Meissner $\mathrm{G}$. Regulation of the cardiac muscle ryanodine receptor by $\mathrm{O}(2)$ tension and $\mathrm{S}$-nitrosoglutathione. Biochemistry. 2008;47(52):13985-13990.

63. Stamler JS, Meissner G. Physiology of nitric oxide in skeletal muscle. Physiol Rev. 2001;81(1):209-237.

64. Bellinger AM, et al. Hypernitrosylated ryanodine receptor calcium release channels are leaky in dystrophic muscle. Nat Med. 2009;15(3):325-330.

65. Durham WJ, et al. RyR1 S-nitrosylation underlies environmental heat stroke and sudden death in Y522S RyR1 knockin mice. Cell. 2008;133(1):53-65.

66. Bellinger AM, et al. Remodeling of ryanodine receptor complex causes "leaky" channels: a molecular mechanism for decreased exercise capacity. Proc Natl Acad Sci U S A. 2008;105(6):2198-2202.

67. Poole DC, Hirai DM, Copp SW, Musch TI. Muscle oxygen transport and utilization in heart failure: implications for exercise (in)tolerance. Am J Physiol Heart Circ Physiol. 2012;302(5):H1050-H1063.

68. Middlekauff HR. Making the case for skeletal myopathy as the major limitation of exercise capacity in heart failure. Circ Heart Fail. 2010;3(4):537-546.
69. Kornberg MD, et al. GAPDH mediates nitrosylation of nuclear proteins. Nat Cell Biol. 2010; 12(11):1094-1100.

70. Colussi C, et al. HDAC2 blockade by nitric oxide and histone deacetylase inhibitors reveals a common target in Duchenne muscular dystrophy treatment. Proc Natl Acad Sci U S A. 2008;105(49):19183-19187.

71. Cacchiarelli D, et al. MicroRNAs involved in molecular circuitries relevant for the Duchenne muscular dystrophy pathogenesis are controlled by the dystrophin/nNOS pathway. Cell Metab. 2010;12(4):341-351.

72. Trivedi CM, et al. Hdac2 regulates the cardiac hypertrophic response by modulating Gsk3 beta activity. Nat Med. 2007;13(3):324-331.

73. Gonzalez DR, Beigi F, Treuer AV, Hare JM. Deficient ryanodine receptor $\mathrm{S}$-nitrosylation increases sarcoplasmic reticulum calcium leak and arrhythmogenesis in cardiomyocytes. Proc Natl Acad Sci U S A. 2007;104(51):20612-20617.

74. Burger DE, et al. Neuronal nitric oxide synthase protects against myocardial infarction-induced ventricular arrhythmia and mortality in mice. Circulation. 2009;120(14):1345-1354.

75. Sun J, Morgan M, Shen RF, Steenbergen C, Murphy E. Preconditioning results in S-nitrosylation of proteins involved in regulation of mitochondrial energetics and calcium transport. Circ Res. 2007;101(11):1155-1163.

76. Clementi E, Brown GC, Feelisch M, Moncada S. Persistent inhibition of cell respiration by nitric oxide: crucial role of S-nitrosylation of mitochondrial complex I and protective action of glutathione. Proc Natl Acad Sci U S A. 1998;95(13):7631-7636.

77. Hare JM, Stamler JS. NO/redox disequilibrium in the failing heart and cardiovascular system. J Clin Invest. 2005;115(3):509-517.

78. Ross JM, Fairchild HM, Weldy J, Guyton AC. Autoregulation of blood flow by oxygen lack. Am J Physiol. 1962;202:21-24.

79. Roach RC, Koskolou MD, Calbet JA, Saltin B. Arterial $\mathrm{O} 2$ content and tension in regulation of cardiac output and leg blood flow during exercise in humans. Am J Physiol. 1999;276(2 pt 2):H438-H445.

80. Gonzalez-Alonso J, Richardson RS, Saltin B. Exercising skeletal muscle blood flow in humans responds to reduction in arterial oxyhaemoglobin, but not to altered free oxygen. J Physiol. 2001;530(pt 2):331-341.

81. Singel DJ, Stamler JS. Chemical physiology of blood flow regulation by red blood cells: the role of nitric oxide and S-nitrosohemoglobin. Annu Rev Physiol. 2005;67:99-145.

82. Doctor A, et al. Hemoglobin conformation couples erythrocyte S-nitrosothiol content to $\mathrm{O} 2$ gradients. Proc Natl Acad Sci U S A. 2005;102(16):5709-5714.

83. Janocha AJ, et al. Nitric oxide during altitude acclimatization. NEngl J Med. 2011;365(20):1942-1944.

84. Sartori C, et al. Selected contribution: long-term effects of beta(2)-adrenergic receptor stimulation on alveolar fluid clearance in mice. J Appl Physiol. 2002;93(5):1875-1880.

85. Deem S, Bishop MJ, Alberts MK. Effect of anemia on intrapulmonary shunt during atelectasis in rabbits. J Appl Physiol. 1995;79(6):1951-1957.

86. Deem S, Swenson ER, Alberts MK, Hedges RG, Bishop MJ. Red-blood-cell augmentation of hypoxic pulmonary vasoconstriction: hematocrit dependence and the importance of nitric oxide. Am J Respir Crit Care Med. 1998;157(4 pt 1):1181-1186.

87. Uncles DR, Daugherty MO, Frank DU, Roos CM, Rich GF. Nitric oxide modulation of pulmonary vascular resistance is red blood cell dependent in isolated rat lungs. Anesth Analg. 1996;83(6):1212-1217.

88. Weissmann N, Grimminger F, Walmrath D, Seeger W. Hypoxic vasoconstriction in buffer-perfused rabbit lungs. Respir Physiol. 1995;100(2):159-169.

89. Han TH, Pelling A, Jeon TJ, Gimzewski JK, Liao JC. Erythrocyte nitric oxide transport reduced by a submembrane cytoskeletal barrier. Biochim Biophys Acta. 2005;1723(1-3):135-142.

90. Han TH, et al. Regulation of nitric oxide consumption by hypoxic red blood cells. Proc Natl Acad Sci U S A. 2003;100(21):12504-12509.

91. Huang KT, Huang Z, Kim-Shapiro DB. Nitric oxide red blood cell membrane permeability at high and low oxygen tension. Nitric Oxide. 2007;16(2):209-216.

92. Jourd'heuil D, Gray L, Grisham MB. S-nitrosothiol formation in blood of lipopolysaccharide-treated rats. Biochem Biophys Res Commun. 2000;273(1):22-26.

93. Crawford JH, Chacko BK, Pruitt HM, Piknova B, Hogg N, Patel RP. Transduction of NO-bioactivity by the red blood cell in sepsis: novel mechanisms of vasodilation during acute inflammatory disease. Blood. 2004;104(5):1375-1382.

94. Deem S, et al. Pulmonary vascular effects of red blood cells containing S-nitrosated hemoglobin. Am J Physiol Heart Circ Physiol. 2004;287(6):H2561-H2568.

95. Crawford JH, et al. Hypoxia, red blood cells, and nitrite regulate $\mathrm{NO}$-dependent hypoxic vasodilation. Blood. 2006;107(2):566-574.

96. McMahon TJ, et al. A nitric oxide processing defect of red blood cells created by hypoxia: deficiency of S-nitrosohemoglobin in pulmonary hypertension. Proc Natl Acad Sci US A. 2005;102(41):14801-14806.

97. Stevenson LW. Design of therapy for advanced heart failure. Eur J Heart Fail. 2005;7(3):323-331.

98. Datta B, et al. Red blood cell nitric oxide as an endocrine vasoregulator: a potential role in congestive heart failure. Circulation. 2004;109(11):1339-1342.

99. Gow AJ, Chen Q, Hess DT, Day BJ, Ischiropoulos H, Stamler JS. Basal and stimulated protein S-nitrosylation in multiple cell types and tissues. J Biol Chem. 2002;277(12):9637-9640.

100. Kluge I, Gutteck-Amsler U, Zollinger M, Do KQ. S-nitrosoglutathione in rat cerebellum: identification and quantification by liquid chromatography-mass spectrometry. J Neurochem. 1997;69(6):2599-2607.

101.Lipton AJ, Johnson MA, Macdonald T, Lieberman MW, Gozal D, Gaston B. S-nitrosothiols signal the ventilatory response to hypoxia. Nature. 2001;413(6852):171-174.

102.Gaston B, et al. Endogenous nitrogen oxides and bronchodilator S-nitrosothiols in human airways. Proc Natl Acad Sci U S A. 1993;90(23):10957-10961.

103. Gaston B, Singel D, Doctor A, Stamler JS. S-nitrosothiol signaling in respiratory biology. Am J Respir Crit Care Med. 2006;173(11):1186-1193.

104. Kasai T, Bradley TD. Obstructive sleep apnea and heart failure: pathophysiologic and therapeutic implications. J Am Coll Cardiol. 2011;57(2):119-127.

105. Wong E, Selig S, Hare DL. Respiratory muscle dysfunction and training in chronic heart failure. Heart Lung Circ. 2011;20(5):289-294.

106.Li F, et al. Regulation of HIF-1alpha stability through S-nitrosylation. Mol Cell. 2007;26(1):63-74.

107. Palmer LA, et al. S-nitrosothiols signal hypoxia-mimetic vascular pathology. J Clin Invest. 2007;117(9):2592-2601.

108. Chowdhury R, et al. Studies on the reaction of nitric oxide with the hypoxia-inducible factor prolyl hydroxylase domain 2 (EGLN1). J Mol Biol. 2011;410(2):268-279.

109.Berchner-Pfannschmidt U, Yamac H, Trinidad B, Fandrey J. Nitric oxide modulates oxygen sensing by hypoxia-inducible factor 1 -dependent induction of prolyl hydroxylase 2. J Biol Chem. 2007;282(3):1788-1796.

110.Tsui AK, et al. Priming of hypoxia-inducible factor by neuronal nitric oxide synthase is essential for adaptive responses to severe anemia. Proc Natl Acad Sci U S A. 2011;108(42):17544-17549.

111.Rao SV, et al. Impact of bleeding severity on clinical outcomes among patients with acute coronary syndromes. Am J Cardiol. 2005;96(9):1200-1206.

112. Kinnaird TD, et al. Incidence, predictors, and prognostic implications of bleeding and blood transfu- 
sion following percutaneous coronary interventions. Am J Cardiol. 2003;92(8):930-935.

113. Wu WC, Rathore SS, Wang Y, Radford MJ, Krumholz HM. Blood transfusion in elderly patients with acute myocardial infarction. $N$ Engl J Med. 2001;345(17):1230-1236.

114.van Veldhuisen DJ, Anker SD, Ponikowski P, Macdougall IC. Anemia and iron deficiency in heart failure: mechanisms and therapeutic approaches. Nat Rev Cardiol. 2011;8(9):485-493.

115. Taylor AL, et al. Combination of isosorbide dinitrate and hydralazine in blacks with heart failure. NEngl J Med. 2004;351(20):2049-2057.

116. Cohn JN, et al. Effect of vasodilator therapy on mortality in chronic congestive heart failure. Results of a Veterans Administration Cooperative Study. N Engl J Med. 1986;314(24):1547-1552.

117. Maroko PR, Braunwald E. Effects of metabolic and pharmacologic interventions on myocardial infarct size following coronary occlusion. Acta Med Scand Suppl. 1976;587:125-136.
118. Borer JS, et al. Reduction in myocardial ischemia with nitroglycerin or nitroglycerin plus phenylephrine administered during acute myocardial infarction. NEngl J Med. 1975;293(20):1008-1012.

119. Rao SV, et al. Relationship of blood transfusion and clinical outcomes in patients with acute coronary syndromes. JAMA. 2004;292(13):1555-1562.

120. Hill SR, et al. Transfusion thresholds and other strategies for guiding allogeneic red blood cell transfusion. Cochrane Database Syst Rev. 2002; (2):CD002042.

121. Murphy GJ, Reeves BC, Rogers CA, Rizvi SI, Culliford L, Angelini GD. Increased mortality, postoperative morbidity, and cost after red blood cell transfusion in patients having cardiac surgery. Circulation. 2007;116(22):2544-2552.

122. Kim P, Dixon S, Eisenbrey AB, O’Malley B, Boura J, O'Neill W. Impact of acute blood loss anemia and red blood cell transfusion on mortality after percutaneous coronary intervention. Clin Cardiol. 2007;30(10 suppl 2):II35-II43.
123. Reynolds JD, Ahearn GS, Angelo M, Zhang J, Cobb F, Stamler JS. S-nitrosohemoglobin deficiency: a mechanism for loss of physiological activity in banked blood. Proc Natl Acad Sci U S A. 2007;104(43):17058-17062.

124.Bennett-Guerrero E, et al. Evolution of adverse changes in stored RBCs. Proc Natl Acad Sci U S A. 2007;104(43):17063-17068

125. Kiraly LN, Underwood S, Differding JA, Schreiber MA. Transfusion of aged packed red blood cells results in decreased tissue oxygenation in critically injured trauma patients. J Trauma. 2009; 67(1):29-32.

126.Shimazutsu K, et al. Inclusion of a nitric oxide congener in the insufflation gas repletes S-nitrosohemoglobin and stabilizes physiologic status during prolonged carbon dioxide pneumoperitoneum. Clin Transl Sci. 2009;2(6):405-412.

127. Metzen E, et al. Nitric oxide impairs normoxic degradation of HIF-1alpha by inhibition of prolyl hydroxylases. Mol Biol Cell. 2003;14(8):3470-3481. 